\title{
Emden's Polytropes: Gas Globes In Hydrostatic Equilibrium
}

\author{
M N Anandaram*
}

\section{Abstract}

The theory of polytropes dealing with the hydrostatic equilibrium structure of gas globes had its origin in Emden's publication, Gaskugeln a century ago (1907). This review article has been written for students of physics and astrophysics not only to understand the theory of polytropes as the simplest of stellar models but also computationally solve the Lane-Emden equation for polytropes. Anyone can easily obtain values of normalized temperature, density, pressure and mass distribution as a function of the normalized radius or mass in any polytrope model in tabular form as well as in graphical form using the program code. Explanation of the algorithm to write a code is provided (python script on request). A graphical description of how the polytropic index determines the structure of the polytrope is also given.

Keywords: Lane-Emden polytropes, gas globes, hydrostatic equilibrium, normalized properties, all-in-one graphs, computer algorithm, analysis.

\section{Introduction}

Polytropes are massive self-gravitating gas globes (spheres). They are models of the simplest possible stellar structure because they are governed by the equations of hydrostatic equilibrium and mass conservation. The equation of hydrostatic equilibrium is given by,

\footnotetext{
* Department of Physics, Bangalore University, Bangalore 560056, India. mnanandaram@gmail.com
} 


$$
\frac{d P(r)}{d r}=-\frac{G M(r)}{r^{2}} \rho(r)
$$

where, $M(r)$ is the mass of an interior sphere of radius $r, \rho(r)$ is the local density and $P(r)$ is the local gas pressure at a distance $r$ from the centre of a gas sphere of total mass $M$ and radius $R$. The equation of mass continuity is given by,

$$
\frac{d M(r)}{d r}=4 \pi r^{2} \rho(r)
$$

where, $d M(r)$ is the mass of a spherical shell of thickness $d r$ surrounding the mass $M(r)$ of radius $r$. We can now build up the model, that is, obtain a run of values of the pressure $P(r)$ and the mass $M(r)$ at any radius $r$ from the centre $(r=0)$ of the polytrope to its surface $(r=R)$ by noting that if $d r$ is the step size then $P(r+d r)=$ $P(r)+d P(r)$ and $M(r+d r)=M(r)+d M(r)$. To do this we need to know the third unknown parameter, the local density $\rho(r)$ from a defined pressure-density relationship.

Emden introduced a polytropic pressure-density relation similar to the equation of state for adiabatic processes [1]. This relation is based on the concept of quasistatic thermodynamic changes of state of an ideal gas during which a generalized specific heat is held constant. Chandrasekhar provides a detailed account of the polytropic changes of state [2].

The polytropic pressure-density relation is defined by the expression,

$$
P \equiv K \rho^{\gamma^{\prime}}=K \rho^{(n+1) / n}
$$

Here $K$ is a constant, $\gamma^{\prime}$ is called the polytropic exponent, and $n$ is called the polytropic index that characterizes the polytrope with a particular pressure- density relationship. Usually the constant $K$ can be found from the physics of the problem (see the Appendix for some comments). The polytropic gas globe models of interest are those with $n=1.5$ (or, $\gamma^{\prime}=5 / 3$ ) which corresponds to adiabatic convective processes, and those with $n=3$ (or, $\gamma^{\prime}=4 / 3$ ) which corresponds to a constant radiation pressure in them. The polytrope with $n=3$ is also known as the Eddington standard 
model, since Eddington used this model to investigate properties of stars in radiative equilibrium. Similarly a polytrope of $n=1.5$ can also describe a low mass white dwarf star supported by the pressure of degenerate nonrelativistic electrons. But if the electrons are fully relativistic then a polytrope of $n=3$ is required to model the white dwarf and obtain its mass known as the Chandrasekhar limit. We can now combine the three equations to derive a dimensionless equation called the Lane-Emden equation. First, equations (1) and (2) are combined to yield,

$$
\frac{1}{r^{2}} \frac{d}{d r}\left(\frac{r^{2}}{\rho} \frac{d P}{d r}\right)=-4 \pi G \rho
$$

This is a form of Poisson's equation for the mass distribution under gravity. Using equation (3), equation (4) becomes,

$$
\frac{1}{r^{2}} \frac{d}{d r}\left[r^{2} K\left(1+\frac{1}{n}\right) \rho^{\frac{1}{n}-1} \frac{d \rho}{d r}\right]=-4 \pi G \rho
$$

Now we shall define a dimensionless variable $\theta$ which relates the local density $\rho(r)$ to the central density $\rho_{c}$ by,

$$
\rho(r)=\rho_{c} \theta^{n}
$$

When $\theta=1, \rho=\rho_{c}$, the central density. So, $\theta=1$ corresponds to $\mathrm{r}=$ 0 the centre of the polytrope. When $\theta=0, \rho=0$, the surface density. So, $\theta=0$ corresponds to $r=R$, the radius of the polytrope. On substituting equation (6), equation (5) becomes,

$$
\alpha_{n}^{2} \frac{1}{r^{2}} \frac{d}{d r}\left(r^{2} \frac{d \theta}{d r}\right)=-\theta^{n}
$$

Here, the radius scale constant $\alpha_{n}$ is defined by,

$$
\alpha_{n} \equiv\left[\frac{(n+1) K}{4 \pi G} \rho_{c}^{\left(\frac{1-n}{n}\right)}\right]^{1 / 2}=\left[\frac{(n+1) P_{c}}{4 \pi G \rho_{c}^{2}}\right]^{1 / 2}
$$

In the last square bracket, $\mathrm{K}$ was eliminated using equation (3) applied to the central pressure and the central density respectively. 
Also, in order to eliminate $\alpha_{n}$ in equation (7), we define a dimensionless radius variable $\xi$, also referred to as the Emden radius, by

$$
\xi \equiv r / \alpha_{n}
$$

Substituting $r=\alpha_{n} \xi$ and $d r=\alpha_{n} d \xi$ equation (7) simplifies to,

$$
\frac{1}{\xi^{2}} \frac{d}{d \xi}\left(\xi^{2} \frac{d \theta}{d \xi}\right)=-\theta^{n}
$$

This is the Lane-Emden equation for index $\boldsymbol{n}$. This expression is a second order differential equation and can also be written as,

$$
\theta^{\prime \prime}=-\theta^{n}-\frac{2}{\xi} \theta^{\prime}
$$

The solution of the Lane-Emden equation, $\theta(\xi)$ known as the LaneEmden function of index $\mathrm{n}$ is obtained by applying an even power series in $\xi$ given by,

$$
\theta(\xi)=\sum_{i=0}^{\infty} a_{i} \xi^{2 i}
$$

Since we know from equation (6) that $\theta(\xi)=1$ at the centre of the polytrope $(r=\xi=0)$, we find immediately here that $a_{0}=1$. When the other six coefficients $a_{i}$ are determined (David Reiss shows the use of the Mathematica software at www.scientificarts.com), the Lane-Emden function is found to be given by the series,

$$
\begin{aligned}
\theta_{n}(\xi) & =1-\frac{1}{6} \xi^{2}+\frac{n}{120} \xi^{4}-\frac{n(8 n-5)}{15120} \xi^{6}+\frac{n\left(122 n^{2}-183 n+70\right)}{3265920} \xi^{8} \\
- & \frac{n\left(5032 n^{3}-12642 n^{2}+10805 n-3150\right)}{1796256000} \xi^{10} \\
+ & \frac{n\left(183616 n^{4}-663166 n^{3}+915935 n^{2}-574850 n+138600\right)}{840647808000} \xi^{12}-\ldots
\end{aligned}
$$


The reader can easily verify at least the first four coefficients of this series by substituting equation (12) into the Lane-Emden equation (10) and comparing coefficients of identical powers of the scaled radius variable $\xi$. The slope, that is, the first derivative of this expression is given by,

$$
\begin{gathered}
\theta_{n}^{\prime}(\xi)=-\frac{1}{3} \xi+\frac{n}{30} \xi^{3}-\frac{n(8 n-5)}{2520} \xi^{5}+\frac{n\left(122 n^{2}-183 n+70\right)}{408240} \xi^{7} \\
-\frac{n\left(5032 n^{3}-12642 n^{2}+10805 n-3150\right)}{179625600} \xi^{9} \\
+\frac{n\left(183616 n^{4}-663166 n^{3}+915935 n^{2}-574850 n+138600\right)}{70053984000} \xi^{11}+\ldots
\end{gathered}
$$

Since the surface of the polytrope of index $\mathrm{n}$ is reached when $\theta_{n}(\xi)$ $=0$, the first root of equation (13) denoted by $\xi_{1}$ is the corresponding dimensionless radius parameter of the polytrope. Then the physical radius of the polytrope is given by $R=\alpha_{n} \xi_{1}$ where the constant is given by equation (9). The quantity $\theta^{\prime}\left(\xi_{1}\right)$ found by evaluating equation (14) at the radius of the polytrope $\xi=\xi_{1}$ is also required to determine the physical properties of the polytrope.

The first root can be found analytically by solving equation (13) only for $\mathrm{n}=0,1$, and 5 :

$\mathrm{n}=0$ : Equation (13) reduces to $\theta_{0}(\xi)=1+\xi^{2} / 6=0$.

Hence $\xi_{1}=\sqrt{6}=2.44949$

$\mathrm{n}=1$ : Equation (13) reduces to $\theta_{1}(\xi)=(\sin \xi) / \xi=0$.

Hence $\xi_{1}=\pi=3.14159$

$\mathrm{n}=5$ : Equation $(13)$ reduces to $\theta_{5}(\xi)=\left(\sqrt{1+\xi^{2} / 3}\right)^{-1}=0$.

Hence $\xi_{1}=\infty$. 
The polytrope of index 5 has the unphysical property of finite mass but infinite radius (see below) and hence it finds application as a zeroth approximation to a red giant star and as a model of the distribution of stars in globular clusters. For other values of $n$, Roxburgh and Stockman [3] point out that the power series solution is typically very slow to converge and, in fact, only converges over the whole model when $\mathrm{n}<1.9$. Hence a computer program is used to do a numerical solution of the Lane-Emden equation and find the first root. Both $\xi_{1}$, and the product $\left[-\xi_{1}^{2} \theta^{\prime}\left(\xi_{1}\right)\right]$ from which $\theta^{\prime}\left(\xi_{1}\right)$ can be found, are listed in Table 1 along with other data.

\begin{tabular}{|l|l|l|l|l|}
\hline \multicolumn{1}{|c|}{$n$} & \multicolumn{1}{|c|}{$\xi_{1}$} & $-\left(\xi^{2} \theta^{\prime}\right)_{\xi=\xi_{1}}$ & \multicolumn{1}{|c|}{$\rho_{c} / \rho$} & $\frac{3}{5-n}=-\Omega /\left(G M^{2} / R\right)$ \\
\hline 0 & 2.4495082 & 4.89909021 & 1.0000000 & 0.600 \\
\hline 0.5 & 2.7526956 & 3.78888395 & 1.8350251 & 0.667 \\
\hline 1.0 & 3.1415926 & 3.14159265 & 3.2898639 & 0.750 \\
\hline $\begin{array}{l}1.5 \\
(\gamma=5 / 3)\end{array}$ & 3.6537499 & 2.71404716 & 5.9907031 & 0.857 \\
\hline 2.0 & 4.3528746 & 2.41104601 & 11.402543 & 1.000 \\
\hline 2.5 & 5.3552754 & 2.18720200 & 23.406462 & 1.200 \\
\hline 3.0 & 6.8968486 & 2.01823595 & 54.182481 & 1.500 \\
$(\gamma=4 / 3)$ & & & & \\
\hline 3.25 & 8.0189375 & 1.94980396 & 88.153242 & 1.714 \\
\hline 3.5 & 9.5358053 & 1.89055709 & 152.88366 & 2.000 \\
\hline 4.0 & 14.971546 & 1.79722991 & 622.40788 & 3.000 \\
\hline 4.5 & 31.836463 & 1.73779887 & 6189.4731 & 6.000 \\
\hline 4.9 & 171.43345 & 1.72461859 & 973805.85 & 30.00 \\
\hline 5 & $\infty$ & 1.73205081 & $\infty$ & $\infty$ \\
\hline
\end{tabular}

Table 1: Physical Parameters of Polytropes (computed from author's python script). 
The central and surface boundary conditions are:

$$
\begin{aligned}
& \text { Centre, }(r=0): \quad \xi=0 ; \theta(0)=1 ; \theta^{\prime}(0)=0 \\
& \text { Surface, }(r=R): \quad \xi=\xi_{1} ; \theta\left(\xi_{1}\right)=0 ; \theta^{\prime}\left(\xi_{1}\right)<0
\end{aligned}
$$

\section{Physical Properties of Polytropes}

We observe from equations (3) and (6) that

$$
\begin{aligned}
& \rho(r)=\rho(\xi)=\rho_{c} \theta^{n} \Rightarrow \theta^{n}=\rho / \rho_{c} \\
& P(r)=P(\xi)=P_{c} \theta^{n+1} \Rightarrow \theta^{n+1}=P / P_{c}
\end{aligned}
$$

The two equations (17) and (18) indicate that once we know the value of $\theta_{n}(\xi)$ we can calculate $\theta^{n}$ and $\theta^{n+1}$ which correspond to the normalized density and normalized pressure respectively at the radius $\xi=r / \alpha_{n}$ in the polytrope. The actual values of the density and pressure can be found after determining their central values. We notice here that $\theta_{n}(\xi)$ it has no defined physical significance.

If we assume that the polytrope contains an ideal gas then it's well known equation of state gives the corresponding temperature as,

$$
T(r)=T(\xi)=T_{c} \theta \Rightarrow \theta=T / T_{c}{ }^{\prime}
$$

where, the central temperature $T_{c}$ is given by

$$
T_{c}=\frac{\mu m_{H}}{k} \frac{P_{c}}{\rho_{c}}
$$

We can now relate the Lane-Emden function $\theta(\xi)$ to the local temperature expressed as a fraction of the central temperature. Thus $\theta, \theta^{n}$ and $\theta^{n+1}$ represent the local values of the normalized temperature, the normalized density and the normalized pressure given by equations (17), (18) and (19) respectively. These three thermodynamic parameters monotonically decrease from unity at the centre to zero at the surface, just as $\theta, \theta^{n}$ and $\theta^{n+1}$ do in the polytrope. We also notice that, the inequality $\theta>\theta^{n}>\theta^{n+1}$ exists 
throughout any polytrope for which $\boldsymbol{n}>\mathbf{1}$, and also since $\theta^{n+1}=\theta^{n} \times \theta$, the following relation should also hold:

$$
\frac{P(\xi)}{P_{c}}=\left(\frac{\rho(\xi)}{\rho_{c}}\right)\left(\frac{T(\xi)}{T_{c}}\right)
$$

Thus a tabular output of $\theta, \theta^{n}$ and $\theta^{n+1}$ can also be included in a computer solution and equation (20) can be verified at any radius.

In the foregoing discussion, we noted that the temperature is coupled to the pressure and the density through the equation of state (19b) which describes an ideal gas that obeys the MaxwellBoltzmann statistics and whose constituent particles are nondegenerate as well as non-relativistic. In the case of some objects like white dwarf stars, neutron stars and red giant star cores the pressure and the density relation becomes independent of the temperature. This is because the hydrostatic pressure is provided by degenerate gases which obey the Fermi-Dirac distribution. In such cases $\theta(\xi)$ is just a parameter from which we can calculate the density and the pressure using equations (17) and (18) respectively. In these objects the conventional temperature may be treated as an absolute zero Kelvin.

We can show that the pressure $P_{e}$ of fully degenerate nonrelativistic electrons is given in terms of atomic constants by,

$$
P_{e}=\frac{h^{2}}{20 m_{e} m_{H}^{5 / 3}}\left(\frac{3}{\pi}\right)^{2 / 3}\left(\frac{\rho}{\mu_{e}}\right)^{5 / 3}=K \rho^{5 / 3}=1.0036 \times 10^{18} \mu_{e}^{-5 / 3} \rho^{5 / 3} \mathrm{Nm}^{-2}
$$

where, $\mu_{e}$, defined as the mean molecular weight per electron has a value 2 for a pure helium white dwarf, $\mathrm{h}$ is the Planck constant, $m_{e}$ is the mass of the electron and $m_{H}$ is the mass of the hydrogen atom and $\rho$ is the local density. When this pressure determines the hydrostatic structure we have a polytrope of index $n=1.5$. On the other hand, if the fully degenerate electrons are also fully relativistic with all electrons moving at the speed of light in the polytrope, we can then show that their pressure is given by, 


$$
P_{e}=\frac{h c}{8 m_{H}^{4 / 3}}\left(\frac{3}{\pi}\right)^{1 / 3}\left(\frac{\rho}{\mu_{e}}\right)^{4 / 3}=K \rho^{4 / 3}=1.2435 \times 10^{19} \mu_{e}^{-4 / 3} \rho^{4 / 3} N^{-2}
$$

Then a polytrope of index $n=3$ can be used to model this case. In fact we can use this expression in equation (26) to show that this polytrope has a total mass given by,

$$
M_{n=3}=\frac{5.82}{\mu_{e}^{2}} M_{s u n} .
$$

For a pure helium white dwarf $\left(\mu_{e}=2\right)$ we immediately obtain the well known Chandrasekhar mass limit of 1.455 solar masses. However, Chandrasekhar also showed that a white dwarf of that mass has a theoretical radius of zero [2].

\section{Radius of a Polytrope}

The radius $R$ of a polytrope of index $n$ can be written from its defining equation (9) as

$$
R=\alpha_{n} \xi_{1}=\left[\frac{(n+1) K}{4 \pi G}\right]^{1 / 2} \rho_{c}^{(1-n) / 2 n} \xi_{1}
$$

\section{Mass of a Polytrope}

The total mass of a polytrope is defined by $M \equiv M(R) \equiv M\left(\xi_{1}\right)$. We first find the mass $M(r) \equiv M(\xi)$ of an interior sphere of radius $r$ given by,

$$
M(r)=\int_{0}^{r} 4 \pi r^{2} \rho(r) d r
$$

Using the definition (9) this becomes,

$$
M(\xi)=4 \pi \alpha_{n}^{3} \rho_{c} \int_{0}^{\xi} \xi^{2} \theta^{n} d \xi
$$

Using equation (10) to replace the expression in the integral, carrying out the integration and evaluating the result using equation (16a) we get, 


$$
M(\xi)=4 \pi \alpha_{n}^{3} \rho_{c}\left[-\xi^{2} \frac{d \theta}{d \xi}\right]_{\xi}=4 \pi \alpha_{n}^{3} \rho_{c}\left[-\xi^{2} \theta^{\prime}\right]
$$

The total mass of the polytrope is then found by evaluating equation (25) at $\xi=\xi_{1}$ :

$$
M_{n}=M\left(\xi_{1}\right)=4 \pi \alpha_{n}^{3} \rho_{c}\left[-\xi^{2} \theta^{\prime}\right]_{\xi_{1}}=4 \pi\left[\frac{(n+1) K}{4 \pi G}\right]^{3 / 2} \rho_{c}^{(3-n) / 2 n}\left[-\xi^{2} \theta^{\prime}\right]_{\xi_{1}}
$$

The increase of mass with radius can be studied by plotting the mass fraction which expresses $M(\xi)$ as a fraction of the total enclosed mass M. It is deduced from equations (25) and (26) as,

$$
M(\xi) / M=\left[-\xi^{2} \theta^{\prime}\right]_{\xi} /\left[-\xi_{1}^{2} \theta^{\prime}\right]_{\xi_{1}}
$$

Where the quantities on the right hand side of the equation can easily be calculated after evaluating the denominator at $\xi=\xi_{1}$.

For the standard model $(n=3)$, we find that its mass $M_{3}$ is independent of its central density from equation (26). To find the total mass for the case $n=5$ since its radius is infinite, we proceed as follows.

We first evaluate the square bracket in equation (26) using equation (16c):

$$
\lim _{\xi \rightarrow \infty}\left[-\xi^{2} \theta^{\prime}\right]=\lim _{\xi \rightarrow \infty}\left[\frac{1}{3} \xi^{3}\left(1+\xi^{2} / 3\right)^{-3 / 2}\right]=\sqrt{3}
$$

The mass fraction for this polytrope is deduced from equation (27) as

$$
\frac{M(\xi)}{M}=\left[\frac{1}{3 \sqrt{3}} \xi^{3}\left(1+\frac{\xi^{2}}{3}\right)^{-3 / 2}\right]
$$

The total mass of the $n=5$ polytrope is found, from equation (26), to be a finite quantity: 


$$
M_{5}=36 \pi\left(\frac{K}{2 \pi G}\right)^{3 / 2} \rho_{c}^{-1 / 5}
$$

\section{Mass-Radius Relationship}

If we invert (24) to get an expression for central density in terms of radius and use it to eliminate it in (26), the resulting $M-R$ relationship would be:

$$
M^{(n-1) / n} R^{(3-n) / n}=\frac{(n+1) K}{(4 \pi)^{1 / n} G}\left[-\xi^{\frac{(n+1)}{(n-1)}} \frac{d \theta}{d \xi}\right]_{\xi=\xi_{1}}^{(n-1) / n}
$$

The right hand side of this expression is always a constant for any given $K$ and $n$. Therefore we can easily deduce from equation (24) that for polytropes with $n=1.5$, the product of their mass and volume is a constant. A consequence is that these polytropes shrink as their mass increases, a result found true for low mass white dwarfs which are supported by the pressure of degenerate non-relativistic electrons. On the other hand equation (24) suggests that the mass of a polytrope with $n=3$ is a constant, and is independent of its radius, a result that leads to the well known limiting mass of white dwarfs deduced by Chandrasekhar.

\section{The Central Condensation}

The ratio of the central density of a polytrope to its mean density is defined as the central condensation. The mean density of a sphere of radius $r=\alpha_{n} \xi$ can be written as,

$$
\bar{\rho}(r)=\frac{3 M(r)}{4 \pi r^{3}}=\overline{\rho(\xi)}=\frac{3 M(\xi)}{4 \pi \alpha_{n}^{3} \xi^{3}}=\frac{3}{\xi^{3}} \rho_{c}\left[-\xi^{2} \theta^{\prime}\right]_{\xi}
$$

where substitution for the mass from (25) has been used. When this is evaluated at the radius of the polytrope, its mean density is obtained as,

$$
\bar{\rho}=3 \rho_{c}\left[-\frac{\theta^{\prime}}{\xi}\right]_{\xi=\xi_{1}}
$$


The central condensation can also be written in a convenient form as,

$$
\frac{\rho_{c}}{\bar{\rho}}=\left[\frac{\xi^{3} / 3}{-\xi^{2} \theta^{\prime}}\right]_{\xi=\xi_{1}}
$$

This parameter is listed in Table 1. It shows that the central density of a polytrope increases as its index value increases, becoming infinite for $n=5$.

The increase in central density also implies that more work is done in compressing matter into the core of the polytrope. This is indicated by the increase in the gravitational binding energy with the polytropic index which is discussed in section 7 and section 8 examines the stability issue. When the mean density $\bar{\rho}$ is expressed in terms of the solar mass, $M_{\text {sun }}=1.9891 \times 10^{30} \mathrm{~kg}$ and the solar radius, $R_{\text {sun }}=6.95508 \times 10^{8} \mathrm{~m}$, and $G=6.67428 \times 10^{-11}$ $\mathrm{m}^{3} \mathrm{~kg}^{-1} \mathrm{~s}^{-2}$ we obtain the following expression:

$$
\rho_{c}=1409\left[\frac{\xi^{3} / 3}{-\xi^{2} \theta^{\prime}}\right]_{\xi=\xi_{1}}\left(\frac{M}{M_{\text {sun }}}\right)\left(\frac{R}{R_{\text {sun }}}\right)^{-3}
$$

\section{The Central Pressure of Polytropes}

The central pressure is given by equation (3a): $P_{c}=K \rho_{c}^{(n+1) / n}$. If we substitute for $K$ and $\rho_{c}$ from equations (30) and (32) respectively, and simplify we get,

$$
P_{c}=\left[4 \pi(n+1)\left(\frac{d \theta}{d \xi}\right)^{2}\right]_{\xi_{1}}^{-1} \frac{G M^{2}}{R^{4}}
$$

If we bring in solar mass and solar radius units equation (33) yields,

$$
P_{c}=\left[\frac{1.126 \times 10^{15}}{4 \pi(n+1)(d \theta / d \xi)_{\xi_{1}}^{2}}\right]\left(\frac{M}{M_{\text {sun }}}\right)^{2}\left(\frac{R}{R_{\text {sun }}}\right)^{-4} \quad N^{-2}
$$




\section{The Gravitational Binding Energy (GBE) of Polytropes}

We now show that the GBE of a polytrope of index $n$, mass $M$ and radius $R$ is given by,

$$
\Omega=-\frac{3}{(5-n)} \frac{G M^{2}}{R}
$$

The total work done in building a spherical polytrope of radius $R$ by adding an incremental mass $d M(r)$ at a time to $M(r)$ at a distance $r$ is given by,

$$
\begin{aligned}
& \Omega=-\int_{0}^{R} \frac{G M(r) d M(r)}{r}=-\frac{G}{2} \int_{0}^{R} \frac{1}{r} d[M(r)]^{2}=-\frac{G}{2}\left\{\frac{M^{2}(r)}{r} \mathfrak{\}_{0}^{R}+\int_{0}^{R} \frac{M^{2}(r)}{r^{2}} d r\right\} \\
& \Omega=-\frac{1}{2} \frac{G M^{2}}{R}-\frac{1}{2} \int_{0}^{R}\left(\frac{G M(r)}{r^{2}} d r\right) M(r) \quad(\because M(0)=0 ; M(R)=M)
\end{aligned}
$$

We now rewrite the equation of hydrostatic equilibrium, equation (1) and show by using the polytropic equation of state equation (3) that

$$
\begin{aligned}
-\left(\frac{G M(r)}{r^{2}} d r\right) & =\frac{d P}{\rho}=(n+1) d\left(\frac{P}{\rho}\right) \\
\because d\left(\frac{P}{\rho}\right) & =\frac{d P}{\rho}-\frac{P}{\rho^{2}} d \rho=\frac{d P}{\rho}-\frac{P}{\rho}\left(\frac{n}{n+1} \frac{d P}{P}\right)=\frac{1}{n+1} \frac{d P}{\rho}
\end{aligned}
$$

Then equation (32a) becomes,

$$
\Omega=-\frac{1}{2} \frac{G M^{2}}{R}+\frac{n+1}{2} \int_{0}^{R} M(r) d\left(\frac{P}{\rho}\right)=-\frac{1}{2} \frac{G M^{2}}{R}-\frac{n+1}{2} \int_{0}^{R} \frac{P}{\rho} d M(r),
$$

since the resulting integrand in the partial integration has vanished. Now we note that $d M(r) / \rho=4 \pi r^{2} d r=d V(r)$ is the corresponding volume of the shell and hence we have, 


$$
\Omega=-\frac{1}{2} \frac{G M^{2}}{R}-\frac{n+1}{2} \int_{0}^{R} P d V(r)
$$

The integral on the right side yields after a partial integration, (the integrand vanishes since at the centre $V=0$ and at the surface $P=$ 0 ) and the use of equations (1) and (2), that,

$$
\begin{aligned}
& \int_{0}^{R} P d V(r)=-\int_{0}^{R} V(r) d P \\
= & -\int_{0}^{R}\left(\frac{4 \pi r^{3}}{3}\right)\left(-\frac{G M(r)}{r^{2}} \rho(r) d r\right)=-\frac{1}{3} \int_{0}^{R}\left(-\frac{G M(r) d M(r)}{r}\right)
\end{aligned}
$$

Recognizing that the last integral is the GBE of the polytrope, we see that,

$$
\int_{0}^{R} P d V(r)=-\frac{1}{3} \Omega
$$

This result also leads to an alternate proof of the virial theorem. We see from this expression that,

$$
-\Omega=3 \int_{0}^{R} P(r) d V(r)=3 \int_{0}^{R} \frac{P(r)}{\rho(r)} d M(r)=3\left(\frac{2}{3} K\right)=2 K,
$$

where we have used the fact that $P / \rho$ is two-third the thermal energy density per unit mass of the nonrelativistic ideal gas, and hence the integral is two-third the total thermal (or, particle kinetic) energy $K$ of the polytrope. So, we get the classical virial theorem, $2 K+\Omega=0$, which has implications for the mean temperature of the polytrope.

Hence using equation (32) in equation (31c) yields,

$$
\Omega=-\frac{1}{2} \frac{G M^{2}}{R}+\frac{n+1}{6} \Omega
$$

This is simplified to the desired result for the GBE of a polytrope of index $n$ : 


$$
\Omega=-\frac{3}{5-n} \frac{G M^{2}}{R}
$$

We notice that $|\Omega|$ increases steadily with $\mathrm{n}$ up to 4.9 and then rapidly increases to become infinite at $n=5$ as shown in Table 1 .

\section{The Internal Energy and the Total Energy of Polytropes}

If we assume that a polytrope contains an ideal gas at a temperature $\mathrm{T}$ and $c_{V}$ is its specific heat at constant volume per unit mass then the internal energy of a mass $\mathrm{dM}(\mathrm{r})$ is $\mathrm{dU}=c_{V}$ $\operatorname{TdM}(\mathrm{r})$ so that its total internal energy is given by,

$$
U=c_{V} \int T d M=c_{V} \int T \rho d V=\frac{1}{(\gamma-1)} \int P d V=-\frac{1}{3(\gamma-1)} \Omega
$$

where we have used the ideal gas equation of state given by,

$$
P=\frac{k}{\mu m_{H}} \rho T=\left(c_{P}-c_{V}\right) \rho T=c_{V}(\gamma-1) \rho T \Rightarrow c_{V} \rho T=\frac{P}{(\gamma-1)}
$$

and also equation (37). The total energy $\mathrm{E}$ of the polytrope is then given by the sum of internal energy and GBE as,

$$
E=U+\Omega=\left(1-\frac{1}{3(\gamma-1)}\right) \Omega=\frac{(\gamma-4 / 3)}{(\gamma-1)} \Omega
$$

In this equation we can replace the adiabatic exponent $\gamma$ by the polytropic exponent $\gamma^{\prime}=(n+1) / n$ and obtain

$$
E=\left(\frac{\gamma^{\prime}-4 / 3}{\gamma^{\prime}-1}\right) \Omega=\left(1-\frac{n}{3}\right) \Omega
$$

Any configuration is stable only when $\Omega<0$ together with $E<0$. This requirement restricts stable polytropes to $\mathrm{n}<3$ from equation (42). Thus an ideal gas polytrope of $n=1.5$ or $\gamma=5 / 3$ is stable with $E=\Omega / 2$. But a polytrope with $n=3$ or $\gamma^{\prime}=4 / 3$ is marginally unstable since $E=0$ for any size. 


\section{Algorithm for Solving the Lane-Emden Equation}

The algorithm required to construct a computer programme may be formulated as follows:

Input data to be supplied:

The desired polytropic index, $\mathbf{n}$.

The step size $\Delta \xi$ set at 0.01 (preferably).

The mass $\mathbf{M}$ and radius $\mathbf{R}$ of the polytrope in solar units.

Output data to be generated:

Calculate, store and tabulate the four quantities $\theta(\xi), \theta^{n}, \theta^{n+1}$,

$-\xi^{2} \theta^{\prime}$ and then the mass fraction $\left[-\xi^{2} \theta^{\prime}\right] /\left[-\xi^{2} \theta^{\prime}\right]_{\xi_{1}}$ up to the radius $\xi_{1}$.

The number of lines in the table is large being equal to $1+\xi_{1} / d \xi$. An interpolation of this data may be carried out to print pruned data corresponding to an exact step size of $\left(\xi / \xi_{1}\right) / 100$. Plot the four quantities $\theta(\xi), \theta^{n}, \theta^{n+1}$ and $\left[-\xi^{2} \theta^{\prime}\right] /\left[-\xi^{2} \theta^{\prime}\right]_{\xi_{1}}$ against the radius fraction $\xi / \xi_{1}$ to produce an all-in-one plot in linear form as well as in semilog form respectively. This is because the linear form of the graph displays the interior details well whereas the semilog form clearly shows the details of the polytropic envelope where the parameters $\theta, \theta^{n}$ and $\theta^{n+1}$ have very low values. It is also instructive to $\operatorname{plot} \theta(\xi), \theta^{n}, \theta^{n+1}$ and the radius fraction $\xi / \xi_{1}$ against the interior mass fraction $\left[-\xi^{2} \theta^{\prime}\right] /\left[-\xi^{2} \theta^{\prime}\right]_{\xi_{1}}$. These may be referred to as all-in-one graphs of normalized parameters remembering that both the plot axes cover a range of values from 0 to 1 only.

\section{Solution Algorithm}

Solve the Lane-Emden equation numerically and compute first the two quantities namely the Lane-Emden function and its slope given by $\theta(\xi)$ and $\theta^{\prime}$ respectively up to the scaled radius $\xi_{1}$. Then compute the density fraction $\theta^{n}$, the pressure fraction $\theta^{n+1}$, the mass 
parameter product $-\xi^{2} \theta^{\prime}$ and the mass fraction $\left[-\xi^{2} \theta^{\prime}\right] /\left[-\xi^{2} \theta^{\prime}\right]_{\xi_{1}}$ up to the radius $\xi_{1}$. Since the Lane-Emden equation is one of second order as was shown in equation (11) it has the form $y^{\prime \prime}=F\left(x, y, y^{\prime}\right)$ where we can identify

$x=\xi, y=\theta, y^{\prime}=\theta^{\prime}=d \theta / d \xi, y^{\prime \prime}=\theta^{\prime \prime}=d^{2} \theta / d \xi^{2}$ and

$F\left(x, y, y^{\prime}\right)=-\frac{2 y^{\prime}}{x}-y^{n}$

The single step self-starting Runge-Kutta $4^{\text {th }}$ order method with truncated error proportional to the $5^{\text {th }}$ power of the step size $\Delta x$ is given by the algorithm (chapter 8, page 273 in Smith [4]):

$$
\begin{aligned}
& y(i+1)=y(i)+\Delta x y^{\prime}(i)+\frac{\Delta x}{6}\left(k_{1}+k_{2}+k_{3}\right), \\
& y^{\prime}(i+1)=y^{\prime}(i)+\frac{1}{6}\left(k_{1}+2 k_{2}+2 k_{3}+k_{4}\right),
\end{aligned}
$$

where the $\boldsymbol{k}^{\prime}$ s are calculated from,

$$
\begin{aligned}
& k_{1}=\Delta x F\left(x(i), y(i), y^{\prime}(i)\right), \\
& k_{2}=\Delta x F\left(x(i)+\frac{\Delta x}{2}, y(i)+\frac{\Delta x}{2}\left(y^{\prime}(i)+\frac{k_{1}}{4}\right), y^{\prime}(i)+\frac{k_{1}}{2}\right), \\
& k_{3}=\Delta x F\left(x(i)+\frac{\Delta x}{2}, y(i)+\frac{\Delta x}{2}\left(y^{\prime}(i)+\frac{k_{1}}{4}\right), y^{\prime}(i)+\frac{k_{2}}{2}\right), \\
& k_{4}=\Delta x F\left(x(i)+\Delta x, y(i)+\Delta x\left(y^{\prime}(i)+\frac{k_{3}}{2}\right), y^{\prime}(i)+k_{3}\right) .
\end{aligned}
$$

The above scheme has the advantage of requiring only six calculations per integration step of a SODE whereas normally ten calculations are required per integration step of the two coupled first order differential equations into which a second order differential equation is converted while maintaining the same accuracy. Begin the first step of the solution at the centre with this assignment: 


$$
\mathrm{i}=1, \xi=0, \theta=1, \theta^{n}=1, \theta^{n+1}=1, h \equiv \theta^{\prime}=0 \text { and } \mathrm{z} 2 \mathrm{~h}=\xi^{2} h=0 .
$$

Then the second step is completed by assignment or calculation as follows:

Set $\xi=d \xi$ and then compute for a small spherical core of this radius,

$$
\begin{aligned}
& \theta=1-\frac{1}{6} \xi^{2}+\frac{n}{120} \xi^{4} \\
& h=-\frac{1}{3} \xi+\frac{n}{30} \xi^{3} ; \\
& z 2 h=\xi^{2} h .
\end{aligned}
$$

Now, from the third step onward an iterative integration using the Runge-Kutta $4^{\text {th }}$ order algorithm is begun under a while loop with the logic condition that $\theta>0$ which yields $d \theta$ and $d h$ at each iteration.

Now update the previous values of $\xi, \theta$ and $h$ by adding their increments and then compute $\theta^{n}, \theta^{n+1}$ and $\xi^{2} h$. This procedure is repeated until the surface is reached.

However, the finite step size makes it impossible to stop the iteration exactly at $\theta=0$ and hence $\theta$ becomes negative. The while loop tests for this zero crossing event continuously at all intermediate steps in each iteration and once it is detected, exits to the previous values at the start of that iteration. Since at this point we still have $\xi<\xi_{1}$ and $\theta>0$ we use the fact that $h \approx \Delta \theta / \Delta \xi=(0-\theta) /\left(\xi_{1}-\xi\right)$ to extrapolate $\xi$ to $\xi_{1}$ (called zeta1 in the programme code) by,

$$
\xi_{1}=\xi-\theta / h
$$

Similarly $h=\theta^{\prime}(\xi)$ is extrapolated to $h_{1}=\theta^{\prime}\left(\xi_{1}\right)$ by using the following result deduced from equation (16b):

$$
h_{1}=h+\left(\xi_{1}-\xi\right)\left(-\frac{2 h}{\xi}-\theta^{n}\right)
$$


(This is called hzeta1 in a programme code.)

Now compute the following final parameters of the polytrope:

$$
\begin{aligned}
& z 2 h 1=\xi^{2} h_{1} ; \\
& \alpha_{n}=R / \xi_{1} ; \quad \text { (in units of Rsun) }
\end{aligned}
$$

D_mean_sun $=1409.0$; (mean density of the sun in SI). Mean density: d_mean $=\bar{\rho}=$ D_mean_sun ${ }^{*} \mathrm{M} / \mathrm{R}^{\wedge} 3$; $\quad$ (in SI units)

Central density:

$$
\rho_{c}=\bar{\rho}\left[\frac{\xi}{-3 \theta^{\prime}}\right]_{\xi=\xi_{1}} \mathrm{~kg} / \mathrm{m}^{3}
$$

Dc $=$ d_mean * (- zeta1 / hzeta1 / 3.0);

Central Pressure:

$$
\begin{gathered}
P_{c}=\frac{8.98 \times 10^{13}}{(n+1)\left[\theta^{\prime}\right]_{\xi_{1}}^{2}} \frac{M^{2}}{R^{4}} \quad \mathrm{~Pa}\left(=N / m^{2}\right) \\
\mathrm{PC}_{\mathrm{C}}=8.98 \mathrm{e} 13 * \mathrm{M}^{\wedge} 2 / \mathrm{R}^{\wedge} 4 /(\mathrm{n}+1) / \text { hzeta }{ }^{\wedge} 2 ;
\end{gathered}
$$

Central Temperature:

Composition: $\mu=1 /(2.0 X+0.75 Y+0.5 Z)$;

$$
T_{c}=2.291 \times 10^{7} \mu\left[\frac{-1}{(n+1) \xi \theta^{\prime}}\right]_{\xi=\xi_{1}} \frac{M}{R} \quad K
$$

Gravitational binding energy:

$$
\mathrm{GBE}=\Omega=-3.8 \times 10^{41} \frac{3}{5-n} \frac{M^{2}}{R} \quad \mathrm{~J}
$$

Code to output the tabulated data and produce all-in-one graphs in both the linear form and in the semi log form is now prepared according to the syntax of the computational software package used. This program has been written in Python script (this can be obtained from the author upon request). Hellings describes a solution using the unavailable QBasic [5] whereas Timmes 
(www.cococubed.com ) provides FORTRAN codes. The plots are discussed in the next section.

The all-in-one plot for the polytrope of index $\mathrm{n}=5$ can be directly produced by noting that, $T / T_{c}=\theta, \rho / \rho_{c}=\theta^{5}$ and $P / P_{c}=\theta^{6}$ where,

$$
\theta(\xi)=\left(\sqrt{1+\xi^{2} / 3}\right)^{-1}
$$

The mass fraction given by equation (28a) can also be included in the plot. This is done by a separate Python script that limits $\xi$ to a large but finite value so that all parameter fractions can be plotted (see Fig. 7 and Fig. 8).

\section{Analysis of the all-in-one Graphs}

The radius dependent all-in-one plots and below each the corresponding mass dependent all-in-one plots are now presented as Fig. 1 for $n=1.5$ to Fig. 8 for $n=5.0$. Again the first three radius dependent all-in-one plots in semilog form are then presented as Fig. 9 for $n=1.5$ to Fig. 11 for $n=4.5$ to show details close to the surface region.

We can begin by taking a look at the curves representing normalized values of $T, \rho, P$ and $M(r)($ or $R(r))$ in these figures. Since the curves represent $\theta, \theta^{n}$ and $\theta^{n+1}$ respectively in each figure, they start with a value of unity at the center and end with zero at the surface. In the middle region of the curves the separation between the curves of $T / T c$ and $P / P c$ increases as index $n$. In fact, the difference between the two parameters, $\theta\left(1-\theta^{n}\right)$ for a given radius, increases with $n$, whereas the difference, $\theta^{n}(1-\theta)$ between $\rho / \rho_{c}$ and $P / P_{c}$, decreases with $n$. This can be gauged in the graphs. We can also observe the increasing slope of the mass fraction curve given by equation (27) in these five figures which indicates increasing compression of matter closer to the center as $n$ increases. These six graphs showing the pattern of alignment of the three curves as a function of the polytropic index may be used as standard reference for analyzing similar all-in-one graphs produced from computed modern stellar models. 
Consider Fig. 1 which describes a wholly convective star $(n=1.5)$. It is observed that the three curves representing $T, \rho$ and $P$ start with a value unity at the center, proceed nearly equally spaced reaching a maximum separation around $0.5 R$, and converge slowly again to vanish at the surface. Both the density variation and the mass distribution are relaxed, that is, they change gradually toward the surface as the central density is just six times the mean density in the convective star. There is thus no pronounced tendency to push and compress matter into the inner parts of convective regions when convection prevails. The tabulated model data of this polytrope indicate that $0.006 \mathrm{M}$ of mass is contained within a radius $0.1 R$ while $0.044 M$ is contained within $0.2 R$.

Now consider the polytrope models shown in Fig. $3(n=3.0)$ to Fig. $8(n=5)$. In Fig. 3, the curve for $\rho$ has moved much closer to that of $P$ and farther away from that of $T$. This shift becomes increasingly more pronounced in each figure with the curves of $\rho$ and $P$ being mass fraction curve is also seen to be rapidly increasing which indicates increase of central density with the polytropic index. Similarly the remaining graphs can be analyzed bearing in mind that for a polytrope of index $n$ the normalized temperature varies as $T / T_{c}=\theta$, the normalized density varies as $\rho / \rho_{c}=\theta^{n}$ and the normalized pressure varies as $P / P_{c}=\theta^{n+1}$.

\section{Conclusion}

The polytropic stellar models are useful in understanding the structure of real stars in a qualitative way to some extent since both polytropes and real stars share the first two equations of stellar structure dealing with hydrostatic equilibrium and mass conservation. However real stars have, in addition, the nuclear energy generation in their cores which starting with their zero age main sequence (ZAMS) stage begin to alter the core chemical composition by gradually increasing the amount of helium and cause stellar evolution at a rate determined by their starting mass and initial chemical composition. Thus real stars only at their ZAMS stage can be compared with polytropes in that they both have uniform chemical composition throughout. Even then the simple polytropic hydrostatic equilibrium has given way to the 
existence of separate zones in convective and radiative equilibriums the extents and locations of which depend on the mass of the star. These regions will have different equivalent polytropic indices and hence single index polytrope models cannot be applied to real stars.

However, an approximation to real stellar models can be constructed by combining or sequencing together polytrope sections of different selected indices. Two examples will be mentioned here. Beech and Mitalas [6], for example, have looked at the effect of varying the core and envelope composition in an $\mathbf{n}=\mathbf{3}$, $\mathbf{n}=\mathbf{3}$ double polytrope model. Eggleton et al. [7] have looked at an analytic double polytrope model with an $\mathbf{n}=5$ core and $\mathbf{n}=\mathbf{1}$ envelope, thus utilizing the analytic solution equations of the LaneEmden equation to describe the effects of having a composition discontinuity at the base of the hydrogen burning shell source. Hellings [5] describes the computation of a composite polytropic model suitable for massive stars (a Python program for doing this is available with the author). All these efforts have limited applicability and the only alternative is to numerically compute full scale evolutionary models which take all the four differential equations of stellar structure into account aided by up to date physics based sophisticated modules for needed constitutive relations and inexpensive but powerful computational power.

Polytropes also continue to be models of first choice for investigating newer phenomena such as radial and transverse oscillations [8-9] and their role in solar and stellar seismology.

Note on figures: The radius fraction dependent and the mass fraction dependent (below) all-in-one plots of parameter fractions are shown for selected polytropic indices in Fig. 1 to 8 . Fig. 9 to 11 show the semilog versions of radius fraction dependent all-in-one plots (shown in Fig. 1, 3 and 5 except Fig. 7) in order to highlight the low values of parameters near the surface of the polytropes. Please note that while the radius fraction has the maximum value of unity the actual value of radius is as given in Table 1. 


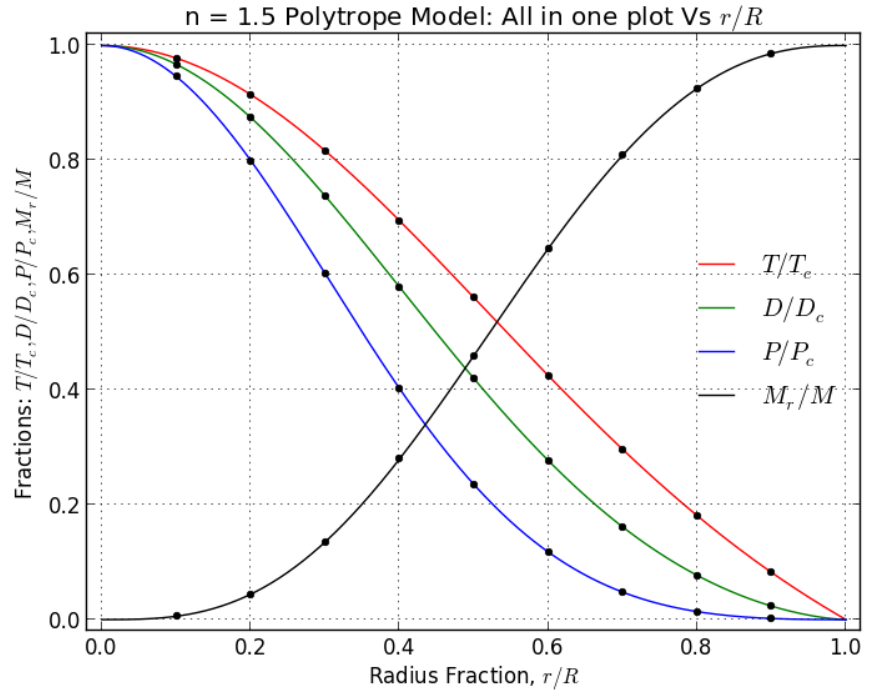

Fig. 1. Radius Fraction $: n=1.5$

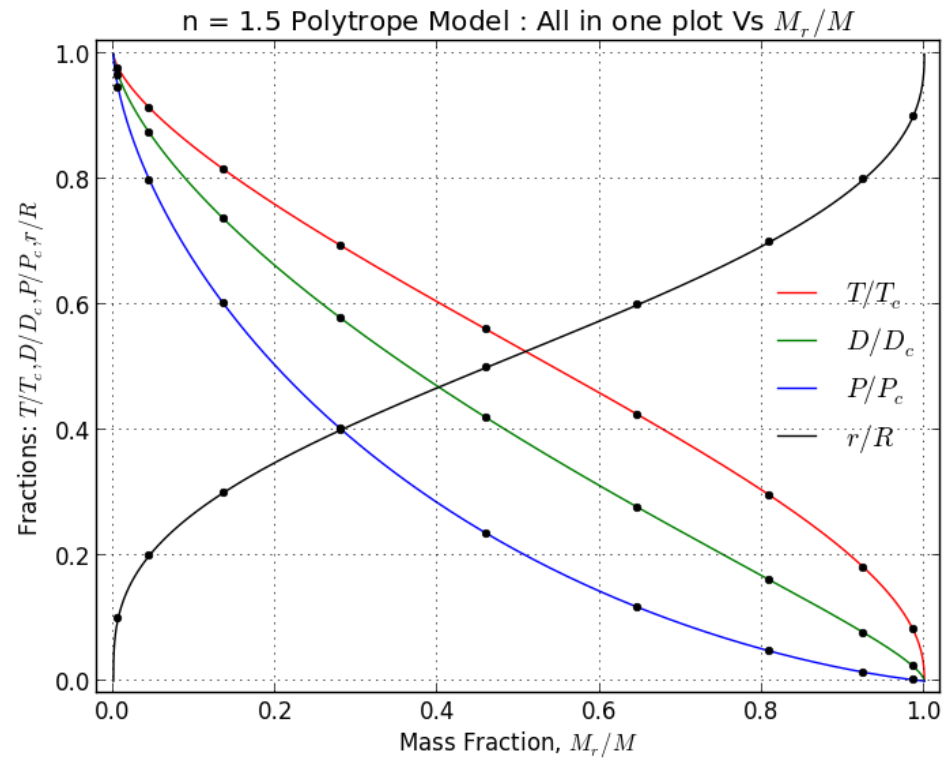

Fig. 2. Mass Fraction: $n=1.5$ 


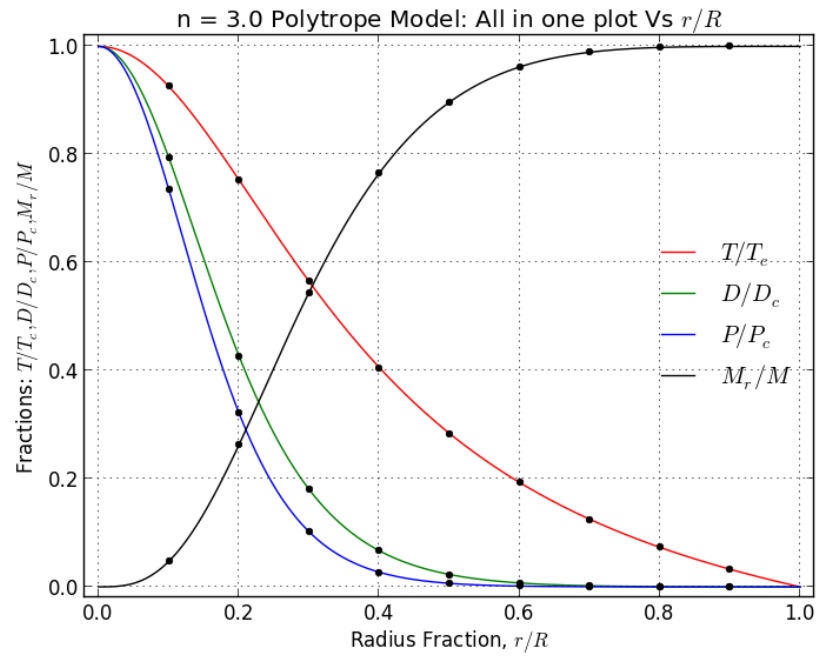

Fig. 3. Radius Fraction: $n=3.0$

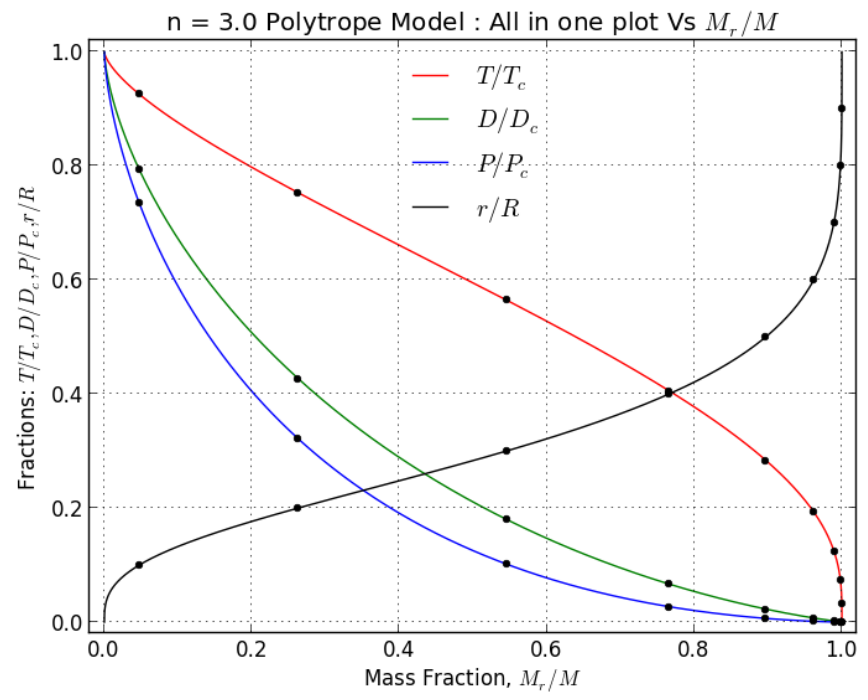

Fig. 4. Mass Fraction: $n=3.0$ 


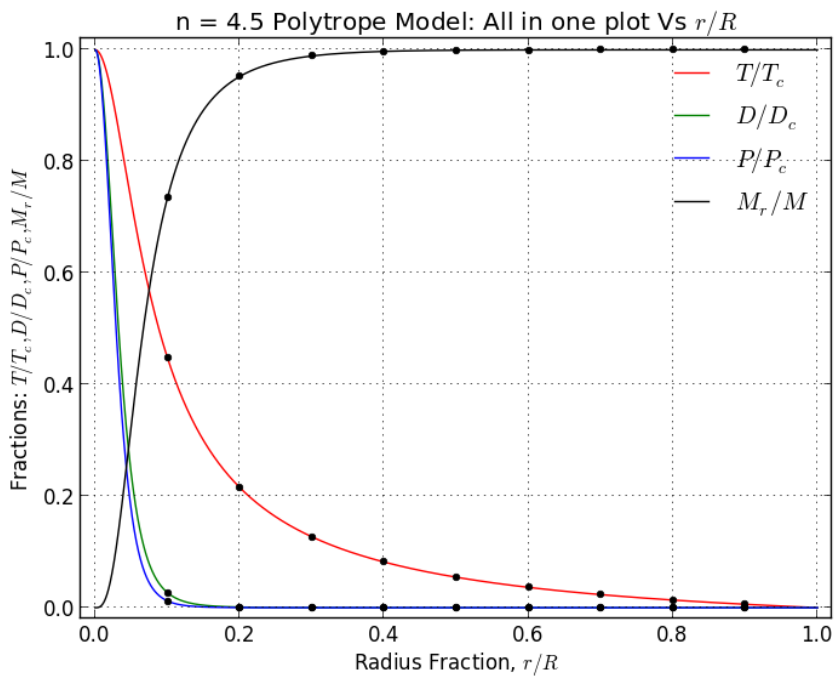

Fig. 5. Radius Fraction: $n=4.5$

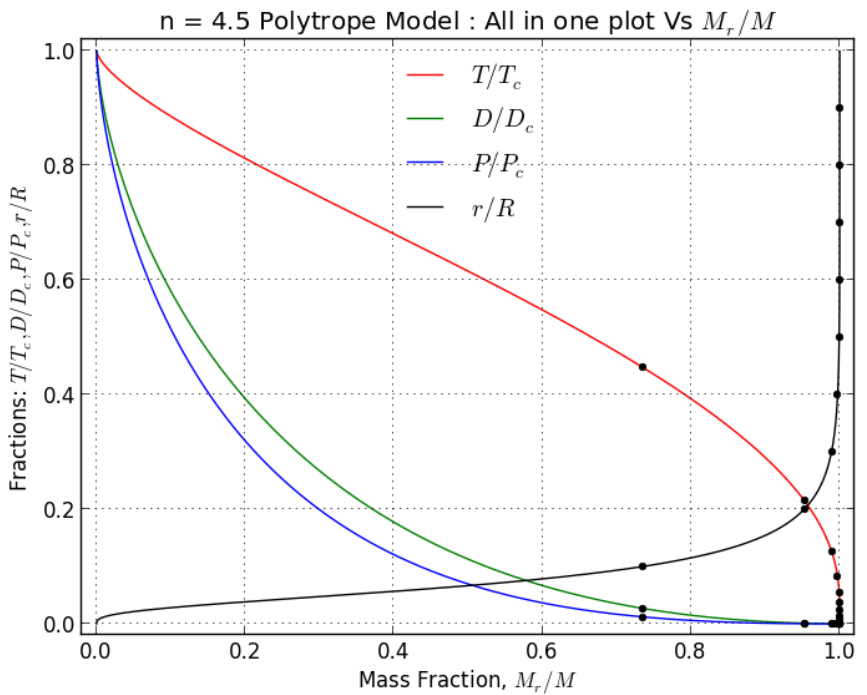

Fig. 6. Mass Fraction: $n=4.5$ 


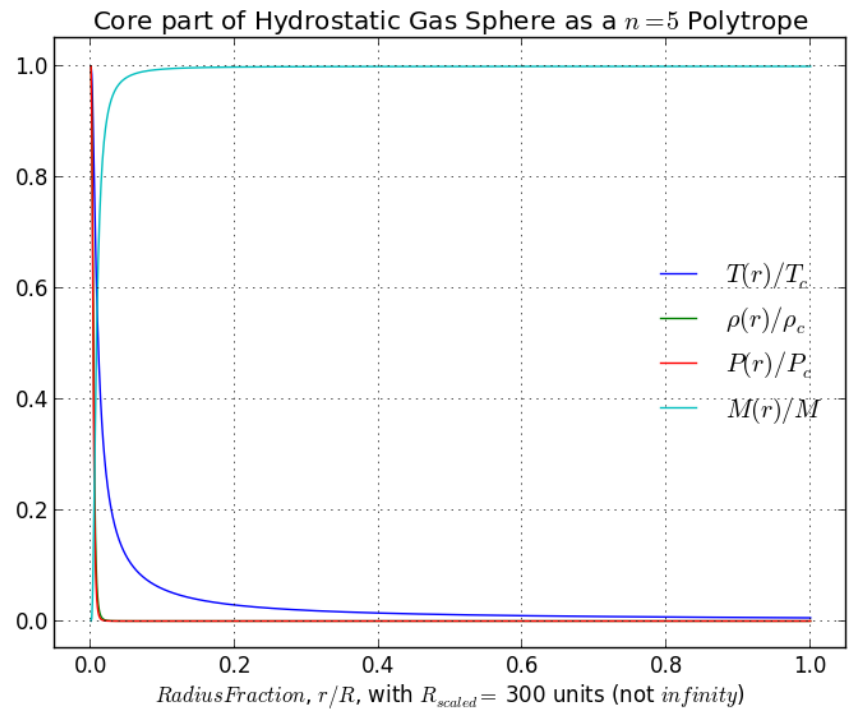

Fig. 7. Radius Fraction (polytropic index $n=5$, core part only)

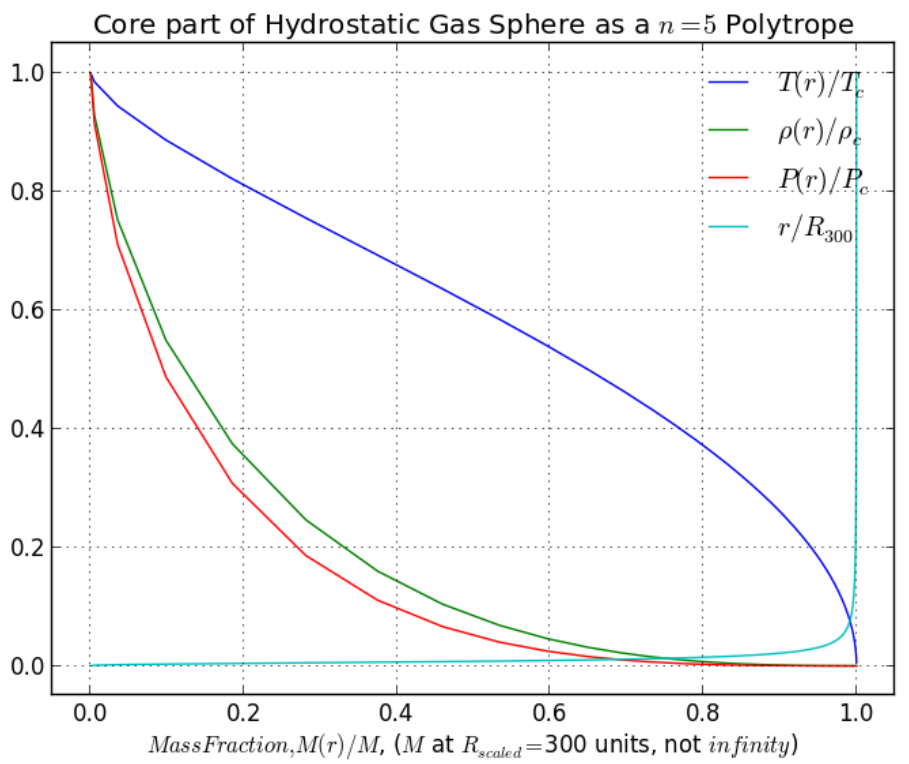

Fig. 8. Mass Fraction (polytropic index $n=5$, core part only) 


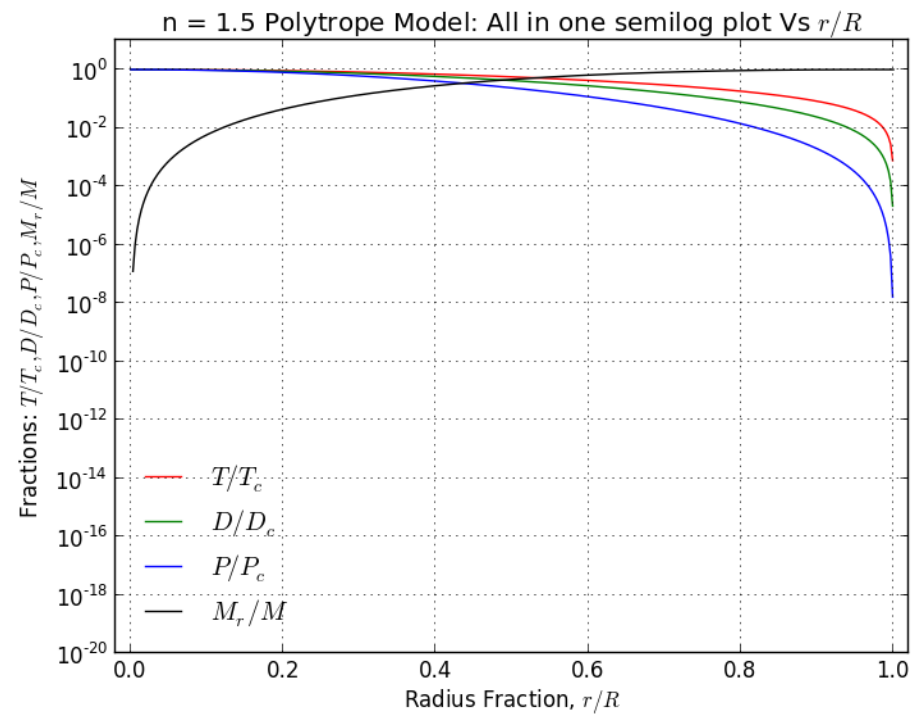

Fig. 9. Semilog Graph: $n=1.5$

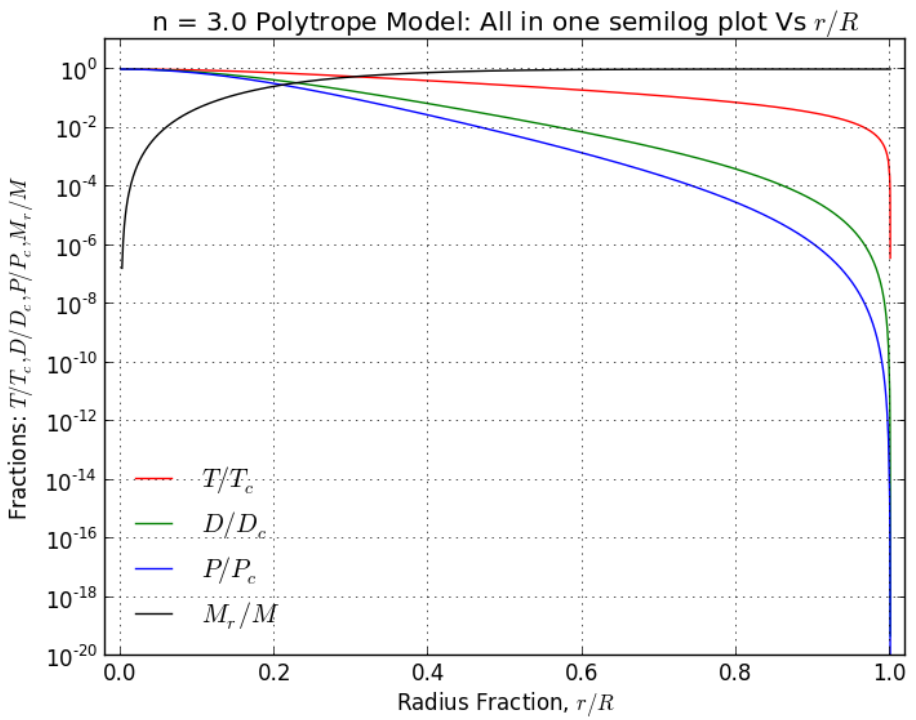

Fig. 10. Semilog Graph: $n=3.0$ 


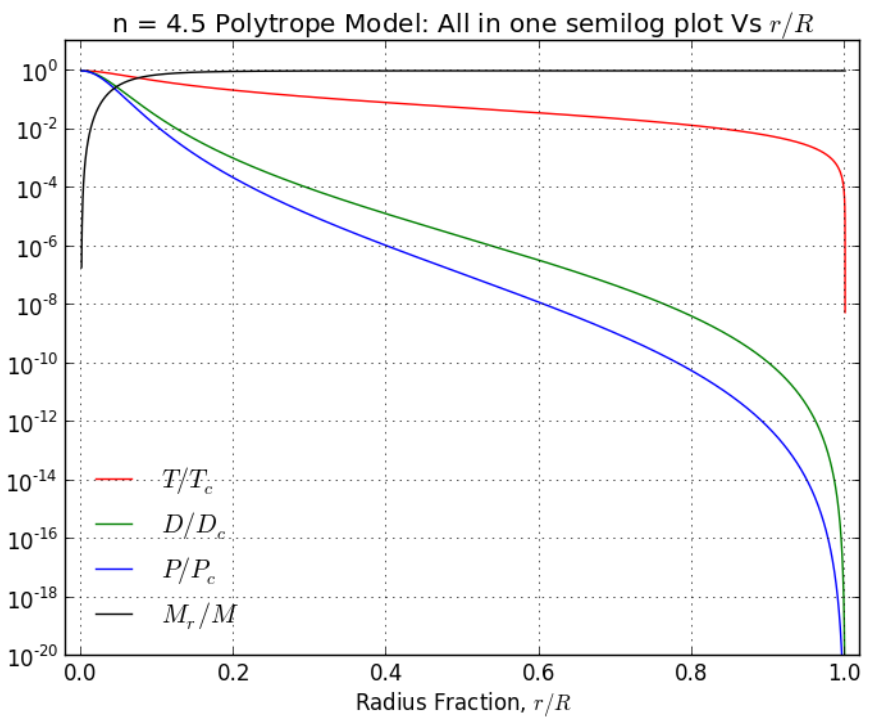

Fig. 11. Semilog Graph: $n=4.5$

\section{Appendix}

This is to draw the attention of the reader to the distinction between the polytropic exponent $\gamma^{\prime}$ and the adiabatic exponent $\gamma$. A polytropic change has $c=d Q / d T=T d S / d T=$ const . But an adiabatic change is a particular case with $c=0$ ant constant entropy so that one can show that

$$
\gamma^{\prime}=\frac{\partial \ln P}{\partial \ln V}=\gamma \frac{c_{v}\left(c-c_{p}\right)}{c_{p}\left(c-c_{v}\right)}
$$

where the adiabatic exponent $\gamma=(\partial \ln P / \partial \ln V)_{S}=c_{p} / c_{v}$ for an ideal gas. In this case one has $\gamma^{\prime}=\left(c-c_{p}\right) /\left(c-c_{v}\right)$. In the adiabatic case, $c=0$ and $\gamma^{\prime}=\gamma$ regardless of the value of $\gamma$.

Consider a star with non-degenerate gas of number density $\mathrm{N}$ (nuclei+electrons) responsible for gas pressure and also containing radiation pressure. If $\beta=P_{\text {gas }} /\left(P_{\text {gas }}+P_{\text {rad }}\right)$ is the gas pressure fraction of total pressure and is constant throughout the star then one can show that $P=\frac{N}{\mu \beta}\left[\frac{3 N}{\mu \beta a}(1-\beta)\right]^{1 / 3} \rho^{4 / 3}$, and, $T=\left[\frac{3 N}{\mu \beta a}(1-\beta)\right]^{1 / 3} \rho^{1 / 3}$ where the local density is $\rho, \mu$ is the mean molecular weight and $a$ is the radiation 
pressure constant and hence here the polytropic index $n=3$. On the other hand for a star in convective equilibrium the entropy must be a constant and by ignoring any radiation pressure one has $n=3 / 2$ and one can show that the entropy and gas pressure are given by $s=\frac{5}{2}-\ln \left[\frac{\rho N}{\mu}\left(\frac{\hbar^{2}}{2 m T}\right)^{3 / 2}\right]=$ const and $P=\frac{\hbar^{2}}{2 m}\left(\frac{\rho N}{\mu}\right)^{5 / 3} \exp \left(\frac{2}{3} s-\frac{5}{3}\right)=K \rho^{5 / 3}$.

\section{References}

[1] D. J. Mullan and R. K. Ulrich, "Radial and Non-radial Pulsations of Polytropes: High-Precision Eigenfrequencies and the Approach of pModes to Asymptotic Behaviour," ApJ, vol. 331, pp. 1013, 1988.

[2] D. J. Mullan, "Physics of the Sun: A First Course," CRC Press, Boca Raton, 2009.

[3] I. W. Roxburgh and L. M. Stockman, "Power series solutions of the Polytrope Equation," MNRAS, vol. 303, pp. 466-470, 1999. http:/ / dx.doi.org/10.1046/j.1365-8711.1999.02219.x

[4] J. M. Smith, "Mathematical Modelling and Digital Simulation forEngineers and Scientists," John Wiley and Sons, New York, 1977.

[5] M. Beech and R. Mitalas, "Effect of mass loss and overshooting on the width of the main sequence of massive stars," ApJ., vol. 352, pp. 291, 1990.

[6] P. Eggleton, J. Faulkner and R. C. Cannon, "A Small Contribution to the Giant problem," MNRAS, vol. 298, pp. 831, 1998. http:/ / dx.doi.org/10.1046/j.1365-8711.1998.01655.x

[7] P. Hellings, "Astrophysics with A PC: An Introduction to Computational Astrophysics," Willmann-Bell, Richmond, Va, USA, 1994.

[8] R. Emden, “Gaskugeln,” B. G. Teubner, Leipzig, 1907.

[9] S. Chandrasekhar, "An Introduction to the Study of Stellar Structure," University of Chicago, Chicago, USA, 1939. 